\title{
Responding to a measles outbreak in a Pacific island community in western Sydney: community interviews led to church-based immunization clinics
}

\author{
Nicola Scott, ab Salwa Gabriel, ${ }^{c}$ Vicky Sheppeard, ${ }^{d}$ Alisa Peacock, ${ }^{c}$ Caroline Scott, ${ }^{c}$ Kristina Flego, \\ Bradley Forssman ${ }^{e}$ and Holly Seale ${ }^{b}$ \\ Correspondence to Nicola Scott (e-mail: nicolascott1 1@hotmail.com).
}

\begin{abstract}
Introduction: There are large Pacific island communities in western and south-western Sydney, New South Wales, Australia. In 2011 and 2012, measles outbreaks disproportionally affected children and youth within these communities. The objectives of this study were to explore barriers to immunization in a Pacific island community from the perspectives of community members and health professionals and to conduct a pilot programme whereby immunization catch-up clinics were held in a Samoan church in western Sydney.

Methods: Interviews were conducted with Pacific island community members $(N=12)$ and health professionals connected with the Pacific island community $(N=7)$ in 2013. A partnership with a local Samoan church was established to provide an accessible venue for immunization catch-up clinics.

Results: Among the community members there were high levels of belief in the importance of immunization and a positive view regarding the protection offered by immunization. A key barrier reported by community members was being busy and therefore having limited time to get children immunized. The important role of the church within the community was emphasized in the interviews, and as a result, two immunization catch-up clinics were held in a Samoan church in western Sydney. The age range of attendees was 7-33 years. A total of 31 measles, mumps and rubella doses and 19 meningococcal $\mathrm{C}$ doses were given during the two clinics.
\end{abstract}

Discussion: The outcomes of the interviews and the subsequent clinics highlighted the potential of churches as a venue for providing public health interventions such as catch-up immunization.

D espite Australia eliminating the transmission of endemic measles in $2005,{ }^{1}$ sporadic measles outbreaks continue to occur. In New South Wales from 2010 to 2013, there were 323 measles notifications with large outbreaks occurring in both 2011 and 2012. ${ }^{2}$ There were 168 cases in the 2012 measles outbreak in New South Wales, ${ }^{3}$ with most cases occurring in south-western and western Sydney with an overrepresentation among people of Pacific island descent. ${ }^{3}$ The 2011 measles outbreak in western Sydney also disproportionally affected the Pacific island population, with $46 \%$ of the 26 cases being of Pacific island descent. ${ }^{4}$

"Pacific island population" refers to people from the islands of Melanesia, the Federated States of Micronesia and Polynesia, ${ }^{5}$ although populations from these different regions are heterogeneous with diverse cultures, languages and religions. ${ }^{5}$ Australia has sizable Pacific island communities in Sydney, Melbourne and Brisbane. $^{6}$

The Blacktown Local Government Area is the largest in New South Wales by population with 312479 residents ${ }^{7}$ and is multicultural with $38 \%$ of all residents reporting they were born overseas and $37 \%$ of residents speaking a primary language other than English. ${ }^{8}$ Samoan is the fifth most common language spoken at home $^{9}$ and is ranked as the 12 th (4624 people) most common ancestry, followed by Fijian at 13th (4105) and Maori at 15th (3282). ${ }^{10}$ Using ancestry, which is the cultural association and ethnic background of an

\footnotetext{
New South Wales Public Health Officer Training Program, Ministry of Health, New South Wales, Australia.

School of Public Health and Community Medicine, University of New South Wales, New South Wales, Australia.

Western Sydney Local Health District Public Health Unit, New South Wales, Australia.

Health Protection New South Wales, Australia.

Nepean Blue Mountains Local Health District Public Health Unit, New South Wales, Australia.

Submitted: 28 July 2014; Published: 6 April 2015

doi: 10.5365/wpsar.2014.5.3.004
} 
individual going back three generations, provides a more accurate reflection of Pacific island population size than country of birth. ${ }^{6}$

To date, very little research has been undertaken into immunization among Pacific island communities in Australia. New Zealand research has found that children with Pacific island backgrounds have a greater risk of poor health and hospitalization for respiratory infections, skin infections and a higher incidence of infectious diseases such as meningococcal disease and measles than New Zealand Maori or other New Zealand children. ${ }^{11}$ Factors found to be significantly associated with incomplete immunization of Pacific island infants in New Zealand include: (1) maternal birthplace (Pacific island born rather than New Zealand born); (2) parity (more than five children); (3) maternal smoking; and (4) difficulty with transport. ${ }^{12,13}$

As Pacific island children and youth were predominant among the people affected in measles outbreaks in New South Wales in 2011 and 2012, we attempted to increase immunization in this group by: (1) exploring the barriers to immunization in the Pacific island community by conducting interviews with community members; and, (2) conducting a pilot programme whereby two immunization catch-up clinics were held in a Samoan church in western Sydney. This paper describes both of these.

\section{METHODS}

\section{Interviews with Pacific island community members}

\section{Study design and sample}

Semi-structured interviews were conducted in 2013 with Pacific island community members and health professionals who worked with or were connected with the Pacific island community. Health professional participants were recruited through the public health unit. A Pacific island community health worker, with in-depth knowledge of the community and extensive experience with community engagement, assisted with the recruitment of community participants. Eligible participants were Pacific island community members with children recruited through local Pacific island women's support groups and a Pacific island church. A verbal explanation of the study was provided in English or Samoan and written participant information and consent forms were provided in English, Samoan and Tongan. Completed consent forms were returned to the researcher via the community health worker. Approximately $80 \%$ of the people who were provided with a consent form returned them; participants were then contacted by the researcher to arrange an interview.

The interviews aimed to gather information on barriers to immunization and views on proposed pilot immunization catch-up clinics. Questions were asked in English in an open-ended manner to allow for expansion. Interviews were conducted in private and lasted approximately 30 minutes each. Community participants were provided with a voucher as reimbursement for their time.

\section{Data analysis}

The interviews were recorded, transcribed verbatim and analysed thematically using QSR International's NVIVO10 qualitative data analysis software. This was then verified with secondary coding.

Ethics approval was obtained from the University of New South Wales.

\section{Immunization catch-up clinics}

\section{Target population and immunization offered}

The primary target population for the immunization catch-up clinics was Pacific island youth aged 10-19 years, as this was the age group commonly affected in the 2011 and 2012 outbreaks. $^{3,4}$ The Australian Childhood Immunization Register (ACIR) is a national register, established in 1996, that records details of vaccinations given to children under seven years of age who live in Australia. ${ }^{14}$ All children who are registered with Medicare (the Australian universal health insurance scheme) are automatically included on the ACIR unless their parents actively seek their removal. Because $99 \%$ of children are registered with Medicare by the age of 12 months, it can be considered a near complete national record. ${ }^{15}$ As ACIR commenced operations in 1997, older Pacific island youth ( $>19$ years) may not have received or have a record of receiving two doses of measles, mumps and rubella (MMR) vaccine, so this age group was also included as 
a secondary target. As well as MMR vaccine, both target populations were also offered meningococcal $C$ vaccine if it was absent from their records.

\section{Venue and logistics for clinics}

Two immunization catch-up clinics were held in a large Samoan church in western Sydney in 2013. The clinics were advertized through a flyer and word of mouth within the community. The first clinic was held on a rehearsal day for a Samoan festival, which was suggested by the church leader, because many children and youth from the congregation would be present. As the completion of a two-dose vaccine schedule for MMR requires a subsequent dose of MMR to be provided at least four weeks after the first valid dose ${ }^{16}$ the second clinic was held one month after the first clinic.

\section{RESULTS}

\section{Interviews with Pacific island community members}

\section{Participants}

The majority of community participants $(N=12)$ originated from Samoa $(n=10)$ with one participant from the Cook Islands and one from Tonga. Reflective of the recruitment process, the majority of participants were female $(n=11)$. All participants had two children or more with half reporting that their children were born in Australia. Of the children born overseas, the majority were born in New Zealand. One quarter of the participants reported they had grandchildren.

Of the seven health professionals interviewed, three were Samoan, two Tongan and two non-Pacific islanders. At the time of the interviews, the health professionals worked in community health, primary care or tertiary hospital care.

\section{Knowledge of immunization}

Community participants stated that immunization was important and spoke positively about immunization protecting their children and themselves from diseases.

Immunization is good for prevention of diseases coming up, and it is good to have some antibodies against diseases in the future. - community member
All participants were able to name at least two diseases that could be prevented by immunization. The majority of participants said whooping cough, measles or chickenpox, with a few participants providing a more comprehensive list including meningococcal disease and hepatitis.

Participants reported feeling there were minimal risks involved with immunization. One participant described a severe adverse reaction to immunization that her brother had back in the islands; however, she felt that this was not a risk in Australia. Reported benefits of immunization included: preventing disease; facilitating good health; and helping children to be safe, active and happy. There was some acknowledgement of the importance of other people immunizing their children and also reference to costs involved with children getting sick if they are not immunized.

Because I don't want them to be sick. I want them to be healthy and it is more helpful to me cause if they are sick I have to take them to the doctor and spend a lot of money. - community member

\section{Barriers to immunization}

\section{Busy lives}

When asked their current views on barriers to immunization, the majority of participants reported that being busy was a factor, which also leads to them forgetting or having limited time to take their children to the doctor. For some participants, there was no sense of urgency around immunization.

My son, he turned four and I forgot, I had it on my reminder and then forgot again. So I didn't get him done until he was four years and four months. And I kept having it on my reminder. It didn't feel like it was urgent. Just business got in the way and I forgot, I'll do it next week. - community member

\section{Migration}

Missing out on immunization due to migration was raised as an issue by the majority of community participants and health professionals. Community members reported that movement frequently occurs from Samoa to New Zealand and then to Australia. This was also highlighted by the health professionals; one specifically identified that some of the Pacific island families move 
when their children are adolescents and they have missed out on some of their immunizations.

... I think it is just the time when they move over and they get missed that way. - health professional

\section{Communication}

Language barriers and lack of communication were not identified as barriers to immunization for the majority of community participants, although this outcome may have been influenced by the fact that the interviews were conducted in English.

In comparison, all of the health professionals interviewed felt this was a key barrier with misinformation and myths about immunization and low levels of health literacy within the Pacific island community.

Low level of understanding and communication, it's more of an education issue that the people don't really understand and the communication is very difficult. - health professional

\section{Strategies to improve immunization rates}

In contrast to the findings from the community members, health professionals felt it was important to provide information and education about immunization including the risks associated with not being immunized.

\section{Organize interpreters to speak with the group and provide information about the value of vaccination, about the risks of not getting your child vaccinated. - health professional}

Due to previous advice given to the public health unit regarding the importance of church in the Pacific island community, interviewees were asked their views on providing information on immunization through church. Both groups agreed with this approach; however, the positive response from the community members may have been influenced by their links to local churches.

Most Pacific island people go to church. Maybe this is one of the best channels to go through. To remind people, through ministers, because their job is spiritual health as well, they will give out information for the health of their people. community member

I guess more awareness, through church, cause that is where most of them go to. The communities
Table 1. Participants of the immunization catch-up clinics by immunization status and whether vaccine given, western Sydney, Australia, 2013

\begin{tabular}{lcc}
\hline \multicolumn{1}{c}{ Immunization status } & \multicolumn{2}{c}{ Number of people } \\
& Clinic 1 & Clinic 2 \\
\hline $\begin{array}{l}\text { Fully vaccinated for age (no vaccine } \\
\text { required) }\end{array}$ & 27 & 1 \\
Vaccine given & 36 & 14 \\
Not vaccinated & 34 & 4 \\
1 documented dose of MMR & 2 & $10^{*}$ \\
Total number of people attending clinic & 63 & 15 \\
\hline
\end{tabular}

* All received their MMR dose 1 at the first clinic.

MMR, mumps, measles and rubella.

move around the church area, so that's one way to improve the awareness and communication through church. - health professional

Interviewees were asked their views on the use of churches to pilot immunization catch-up clinics. The majority of community members said they would be happy for their children or grandchildren to be immunized at church. All of the health professionals agreed with the approach.

The parents go there and seem to respect the leadership from the ministers, so I think we will have more success that way. - health professional

\section{Immunization catch-up clinics}

Approximately 70 children and youth were in attendance at the first clinic held on a festival rehearsal day. An older age group was in attendance at the second clinic held on a fundraiser day for the church. On both clinic days, completed consent forms were used to check $A C I R$ and school vaccination records to determine the need for MMR or meningococcal $C$ vaccine.

There were 63 participants at the first clinic (approximately 10 people did not consent) and 15 at the second (Table 1). Of these, 27 at the first clinic and one at the second clinic were appropriately vaccinated; a total of 50 doses of vaccine were provided: 36 at the first clinic and 14 at the second (Table 1 ).

Participants already vaccinated with MMR had an age range of $7-17$ years (median $=12$ years), and for 
those that received MMR the age range was 10-29 years (median $=20$ years). For those already vaccinated for meningococcal $\mathrm{C}$, the age range was $7-27$ years (median $=16$ years), and for those provided with meningococcal $\mathrm{C}$ vaccine, the age range was 10-33 years (median = 20 years).

The important role of the church within the community was emphasized in the interviews and as a result, two immunization catch-up clinics were held in a Samoan church in western Sydney.

\section{DISCUSSION}

The Pacific island community in western Sydney has been disproportionally affected by measles outbreaks in recent years. This study indicates that among Pacific island community members interviewed, there were high levels of belief in the importance of immunization and a positive view of protection offered by immunization. A key barrier reported by the community members were being busy and forgetting immunization due to time limitations. Health professionals interviewed felt that a key barrier was low levels of knowledge and health literacy affecting immunization. Both groups agreed that missing out on immunization occurs in the Pacific island community due to migration between countries.

Immunization clinics held in a Samoan church in western Sydney provided an easily accessible venue for church attendees to receive catch-up immunizations. As a pilot programme, gaining consent, checking the registry and providing the required immunizations on the same day was a successful model. However, this approach is resource and time intensive and requires a good relationship with the community. In comparison to a previous Pacific island community-based catch-up clinic held by the same public health unit, where there were no attendees, this church-based clinic immunized a relatively large number of people. This may have been due to factors such as the church venue, that there was a rehearsal for a festival and that the clinics were held on weekends. The median age of people requiring both MMR and meningococcal $C$ vaccine was 20 , suggesting that young adults may require future targeted strategies to improve immunization. Susceptibility of the teenage/ young adult age group to measles has also been seen across New South Wales; 15-19 year olds were the second highest age group affected in the 2012 measles outbreak. $^{3}$

The interviews, although conducted with a small number of Pacific island community members, provided information on knowledge and attitudes towards immunization. This is important as attitudes, perspectives, health beliefs and health-seeking behaviour are shaped by ethnicity and culture ${ }^{17}$ with different cultural groups understanding and experiencing health, health care, disease and treatment differently. ${ }^{17} \mathrm{~A}$ Pacific island and Maori health needs assessment conducted by Queensland Health found that health literacy was very poor among all Pacific island communities; there were low levels of health-seeking behaviour, as well as a lack of knowledge of services and how to navigate the health system. ${ }^{6}$ Traditional beliefs about health were also prevalent, but these beliefs were not discussed in relation to immunization. Churches and religious groups were identified as having a positive influence on social cohesion and health outcomes within the Pacific island community. ${ }^{6}$

Our interviews also highlighted the influence of the church and that it may be a suitable location for catch-up immunization for this high-risk group. To our knowledge, there have been no other reports of immunization clinics being held in churches.

Attitudes and beliefs about immunization in the Pacific island community in Australia may be influenced by experiences in the country of origin. Some Pacific island countries record high incidences of infant and child mortality rates. As well as neonatal causes, other causes of death among children under five include diarrhoeal disease, pneumonia and measles. ${ }^{18}$ Immunization data show varying levels of coverage for measles-containing vaccine (MCV) among Pacific island countries. From 2008 to 2012, MCV coverage ranged from $99 \%$ to $95 \%$ in Tonga, from $95 \%$ to $97 \%$ in Cook Islands, and from $45 \%$ to $85 \%$ in Samoa. ${ }^{19}$ There is inconsistency in coverage rates over the five year period; however Tonga and Cook Islands report they are able to sustain consistently high levels of coverage. The lower MCV coverage rates in Samoa may effect immunization coverage in Samoan communities in Australia. The inconsistencies between Pacific island countries may be due to differences in systems of primary 
health care and immunization service delivery among countries; there appears also to be a knowledge gap that could be addressed with further research.

In Australia, little is known about Pacific island communities' knowledge, attitudes and beliefs towards primary health care services such as immunization. There has been one study of accessibility and utilization of health services conducted in Brisbane in four culturally and linguistically diverse communities in 2011. This found that in the Pacific island community there was lack of knowledge of available health services, reported communication issues due to use of medical terminology by health workers and acknowledgement of a preference for accessing doctors from a similar cultural background. ${ }^{17}$ While our study focused only on immunization, these broader insights could be useful for improving delivery of culturally appropriate primary health care such as immunization clinics in the Pacific island community in New South Wales.

This study had some limitations: interviews were only conducted with a small group of participants; conducting the interviews in English may have resulted in selection bias towards those able to converse in English; detailed socioeconomic and demographic information was not collected; and community participants were linked to the women's group and the church from which they were recruited. This reduces the representativeness to the rest of the Pacific island community and also the possibility that other important emergent themes were not discovered. Limitations of the clinics were that some of the first clinic attendees did not return to receive their second MMR dose and that the clinics focused on one Pacific island community and one religious denomination within that community. Using open-ended questions enabled a greater depth of information and secondary coding verification strengthened the analysis.

\section{CONCLUSIONS}

By interviewing a small number of community members and health professionals, the influence of the church was identified, and, as a result, two immunization clinics were held in a large Samoan church in western Sydney. This alternative approach for providing immunization to most-at-risk group was successful and highlighted that a partnership between a local church and public health unit can be effective in providing catch-up immunization.
However, this approach is resource and time intensive and requires a good relationship with the community to ensure success. Rather than implementing a regular programme, a church or similar community location could be used to target high-risk groups for immunization in outbreak situations.

\section{Conflicts of interest}

None declared.

Funding

Holly Seale is supported by an NHMRC Australian-based Public Health Training Fellowship (1012631).

\section{Acknowledgements}

This work was completed while Nicola Scott was an employee of the New South Wales Public Health Officer Training Program, funded by the New South Wales Ministry of Health, while based at the Western Sydney Public Health Unit.

\section{References:}

1. Heywood $A E$ et al. Elimination of endemic measles transmission in Australia. Bulletin of the World Health Organization, 2009, 87:64-71. doi:10.2471/BLT.07.046375 pmid:19197406

2. Measles notification in NSW residents. Sydney, New South Wales Ministry of Health, 2013 (http://www0.health.nsw.gov.au/data/ diseases/measles.asp, accessed 2 February 2014).

3. Najjar Z et al. Sustained outbreak of measles in New South Wales, 2012: risks for measles elimination in Australia. Western Pacific Surveillance and Response Journal, 2014, 5:14-20. doi:10.5365/wpsar.2013.4.4.001 pmid:25635228

4. Flego K, Sheppeard V, McPhie K. A recent measles outbreak in Western Sydney - diagnosis and population vaccination status. Broad Street Pump, 2011, 23:1-2.

5. White R et al. Ethnic gangs in Australia: do they exist? - Report No. 3 Pacific Islander Young People. Melbourne, Australian Multicultural Foundation, 1999.

6. Queensland Health response to Pacific Islander and Maori Health needs assessment. Brisbane, Queensland Health, Division of the Chief Health Officer, 2011.

7. Demographics. Sydney, Blacktown City Council, 2013 (http:// www.blacktown.nsw.gov.au/Discover_Blacktown/Statistics/ Demographics, accessed 5 March 2015).

8. Blacktown City Council. Birthplace. Sydney, Blacktown City Council, 2013 (http://profile.id.com.au/blacktown/birthplace, accessed 5 March 2015).

9. Language spoken at home. Sydney, Blacktown City Council, 2013 (http://profile.id.com.au/blacktown/language, accessed 5 March 2015).

10. Ancestry. Sydney, Blacktown City Council, 2013 (http://profile. id.com.au/blacktown/ancestry, accessed 5 March 2015). 
11. Tukuitonga CR, Bell S, Robinson E. Hospial admission among Pacific children Auckland 1992-97. The New Zealand Medical Journal, 2000, 113:358-361. pmid:11130369

12. Paterson J et al. Maternal and demographic factors associated with non-immunisation of Pacific infants living in New Zealand. The New Zealand Medical Journal, 2004, 117:U994. pmid: 15475977

13. Paterson $\mathrm{J}$ et al. Immunisation of a cohort Pacific children living in New Zealand over the first 2 years of life. Vaccine, 2006, 24:4883-4889. doi:10.1016/j.vaccine.2006.02.050 pmid: 16644070

14. Australian childhood immunisation register. Canberra, Department of Human Services, 2013 (http://www.medicareaustralia.gov.au/ provider/patients/acir/, accessed 11 February 2014).

15. Hull BP, Deeks SL, McIntyre PB. The Australian Childhood Immunisation Register-A model for universal immunisation registers? Vaccine, 2009, 27:5054-5060. doi:10.1016/j. vaccine.2009.06.056 pmid:19576945

16. The Australian Immunisation Handbook - 10th edition. Canberra, Department of Health and Ageing, 2013.

17. Henderson S, Kendall E. Culturally and linguistically diverse peoples' knowledge of accessibility and utilisation of health services: exploring the need for improvement in health service delivery. Australian Journal of Primary Health, 2011, 17: 195-201. doi:10.1071/PY10065 pmid:21645477

18. Gani A. Some aspects of communicable and non-communicable diseases in Pacific Island countries. Social Indicators Research, 2009, 91:171-187. doi:10.1007/s11205-008-9276-x

19. WHO-UNICEF Estimates of measles containing vaccine (MCV) coverage. Geneva, World Health Organization, 2014 (http://apps. who.int/immunization_monitoring/globalsummary/timeseries/ tswucoveragemcv.html, accessed 5 March 2015). 